\title{
La práctica de valores y su incidencia en la formación profesional de las y los estudiantes de la Facultad de Trabajo Social de la Universidad Laica Eloy Alfaro de Manabí
}
The practice of values and its incidence in the professional training of the students of the Faculty of Social Work of the Laica University Eloy Alfaro de Manabi

\author{
Marjorie M. Gómez-Zambrano ${ }^{\mathrm{I}}$ \\ Universidad Laica "Eloy Alfaro" de Manabí \\ Manta, Ecuador
}

Silvia Del P. Menéndez-Rodríguez II 


\section{Resumen}

Para realizar esta investigación se utilizaron las técnicas de: entrevista, la misma que fue aplicada a la Decana y al primer vocal del Consejo de Facultad, las encuestas dirigidas a los estudiantes y a los docentes de la Facultad de Trabajo Social, el resultado de ambas técnicas permitió conocer la incidencia de la deficiente práctica de valores en la formación profesional. Además se aplicó un registro de observación a los estudiantes (grupo de control y grupo experimental) para conocer la incidencia de los talleres de educación en valores.

Palabras clave: Valores; formación profesional; estudiantes. 


\section{Abstract}

In order to carry out this research, the following techniques were used: interview, the same one that was applied to the Dean and the first member of the Faculty Council, the surveys directed to the students and to the teachers of the Faculty of Social Work, the result of both techniques It allowed to know the incidence of deficient practice of values in professional training. In addition, an observational record was applied to the students (control group and experimental group) to know the incidence of the value education workshops.

Key words: Values; vocational training; students. 


\section{Introducción.}

En los momentos actuales las perspectivas fundamentales en la enseñanza universitaria están basadas en una educación a lo largo de la vida. Las exigencias de la época para todas las profesiones apuntan a la necesidad de elevar la calidad de los procesos formativos tanto en el orden científicotécnico y profesional como también en sus cualidades morales.

"La escuela es el modelo más elevado de la vida social...es el tiempo de la esperanza...la construcción de lo común, desde lo diferente." (Cullen C. 1999)

El proceso de formación de valores no es un fenómeno que cierra en una edad determinada. Hay valores que se incorporan y se reajustan a los principios y convicciones personales en el transcurso de la vida.1

En la Conferencia Mundial sobre Educación Superior (1998): "Visión y acción para el siglo XXI", se subraya el acceso abierto a la enseñanza superior, con una óptima gama de opciones y posibilidades de entrar y salir fácilmente al sistema sin distinción de edad y sin ninguna discriminación que propicie el aprendizaje permanente, con el fin de formar ciudadanos con una calidad no solo en competencia profesional sino en la formación de valores humanos y profesionales que puedan solucionar problemas en la sociedad, con responsabilidad, ética y autonomía.2

Este planteamiento que sustenta las tendencias de desarrollo: Masificación, Diversificación y flexibilidad curricular, las nuevas expectativas de desarrollo en la carrera de Licenciatura en Enfermería, así como los supuestos teóricos, posibilitan la comprensión y orientación de: ¿cómo desarrollar el proceso de enseñanza aprendizaje en la carrera de Enfermería para contribuir a la formación de valores profesionales? ¿Qué concepciones y práctica educativa propicia de manera 
significativa la materialización de estos propósitos formativos? Partiendo de estas interrogantes y sustentado en los referentes teóricos abordados, nos proponemos desde el proceso de enseñanza aprendizaje propiciar la formación y el fortalecimiento de los valores esenciales, de acuerdo con el modelo del profesional y las exigencias de la época y de nuestra sociedad.

Las exigencias de la época y de nuestra sociedad, reclaman de la educación universitaria en Enfermería romper las concepciones tradicionales de la enseñanza, comprender la necesidad de una concepción y práctica educativa interactiva y de influencia mutua, de modo que el estudiante en un ambiente de diálogo, reflexión y participación activa busque, indague, construya sus propios conocimientos, habilidades y valores.

\section{Metodología.}

En la investigación se aplicaron los siguientes métodos:

MÉTODO INDUCTIVO.- Se utilizó en el proceso de la recopilación de la información para realizar el marco teórico y de las técnicas aplicadas, además en la organización de la teoría que sirvió para sustentar la presente investigación.

MÉTODO DEDUCTIVO.- Se lo aplicó en el proceso de la selección de la información más importante de la presente investigación, la cual fue tomada de diversas fuentes bibliográficas y poder de esa manera, redactar las conclusiones.

MÉTODO ESTADíSTICO.- Fue importante para la representación gráfica, se analizaron los resultados de las encuestas aplicadas, los mismos que sirvieron de apoyo para las conclusiones. 
MÉTODO CUALITATIVO.- Sirvió para conocer detalladamente cómo incide la práctica de valores en la formación profesional de los estudiantes, y que constan en las encuestas, además de las fichas de observación que se aplicaron.

MÉTODO CUANTITATIVO.- Una vez que se recopiló la información respectiva, se valoró y presentó con datos a través de los cuadros estadísticos y gráficos.

MÉTODO ANALÍTICO.- Permitió realizar el análisis de los resultados de las encuestas y entrevistas que se aplicaron a los estudiantes, docentes y autoridades.

MÉTODO SINTÉTICO.- Fue utilizado para sintetizar la información y teoría de toda investigación realizada, detallando solo lo más importante y redactar las conclusiones.

MÉTODO PROPOSITIVO.- Al final de la investigación fue útil para plantear una propuesta de solución al problema detectado, que beneficie a los estudiantes de la Facultad de Trabajo Social.

\section{TÉCNICAS}

Para realizar esta investigación se utilizaron las técnicas de: entrevista, la misma que fue aplicada a la Decana y al primer vocal del Consejo de Facultad, las encuestas dirigidas a los estudiantes y a los docentes de la Facultad de Trabajo Social, el resultado de ambas técnicas permitió conocer la incidencia de la deficiente práctica de valores en la formación profesional. Además se aplicó un registro de observación a los estudiantes (grupo de control y grupo experimental) para conocer la incidencia de los talleres de educación en valores. 


\section{POBLACIÓN Y MUESTRA}

\section{POBLACIÓN O UNIVERSO}

La población o Universo que se tomó en cuenta para esta investigación estuvo conformada por 36 estudiantes, 20 docentes de la Facultad de Trabajo Social de la Universidad Laica Eloy Alfaro de Manabí.

\section{MUESTRA}

La muestra que se escogió en esta investigación es la probabilística aleatoria para lograr que todos los que conforman el universo tengan la posibilidad de participar, sin ningún tipo de criterio.

\section{TAMAÑO DE LA MUESTRA}

36 estudiantes

\section{APLICACIÓN DE LA FÓRMULA}

$$
\begin{aligned}
& \mathrm{n}=\frac{Z^{2} P Q N}{Z^{2} P Q+N e^{2}} \\
& \mathrm{n}=\text { Tamaño de la muestra } \\
& \mathrm{P}=\text { Probabilidad de ocurrencia } \\
& \mathrm{Q}=\text { Probabilidad de no ocurrencia } \\
& \mathrm{N}=\text { Población } \\
& \mathrm{Z}=\text { Nivel de confianza (entre } 0,95 \text { ) } \\
& \mathrm{e}=\text { Nivel de significancia (admisible ente } 0,5 \text { ) }
\end{aligned}
$$




\section{DESCRIPCIÓN DEL PROCESO DE RECOLECCIÓN DE LA INFORMACIÓN}

Para la realización de las encuestas se contó con la autorización de la Decana de la Facultad de Trabajo Social. La recopilación de la información se la realizó a través de la investigación de Campo, se aplicó una encuesta a los estudiantes para conocer cómo incide la práctica de valores en su formación profesional; a los docentes para conocer cuál es el compromiso que ellos asumen en la práctica de los valores y coadyuvar en la formación profesional de los estudiantes; Una entrevista a los Directivos y Autoridades para conocer cuál es el nivel de aplicabilidad de los valores; Además se aplicó un Registro de Observación para conocer las actitudes de los estudiantes involucrados antes y después de los talleres de valores aplicados.

\section{PROCESAMIENTO DE LA INFORMACIÓN}

Para realizar el procesamiento de la información se utilizó software de aplicación como Word, Excel, Project y Power Point. Cada uno para una tarea específica.

Word se lo utilizó para digitar el contenido, con Excel para realizar los respectivos cálculos estadísticos, verificación de la hipótesis y elaboración del presupuesto. Project. Se lo utilizó para elaborar el cronograma de actividades y Power Point. Se lo utilizó para diseñar las diapositivas a utilizar en la defensa de esta investigación. 
La práctica de valores y su incidencia en la formación profesional de las y los estudiantes de la Facultad de Trabajo Social de la Universidad Laica Eloy Alfaro de Manabí

\section{Resultados.}

\begin{tabular}{|c|c|c|c|c|c|}
\hline OBJETIVOS & TECNICAS APLICADAS & ITEMS & ALTERNATIVAS & & OS \\
\hline & & & & $\mathrm{F}$ & $\%$ \\
\hline OBJETIVO & Encuesta dirigida a las y los & 1. ¿La práctica de los valores es & A. $\mathrm{Si}$ & 125 & $83 \%$ \\
\hline ESPECIFICO $\mathrm{N}^{\circ} 1$ & Estudiantes & $\begin{array}{l}\text { parte de su formación Profesional en } \\
\text { la Facultad de Trabajo Social? }\end{array}$ & B. No & 25 & $17 \%$ \\
\hline Identificar el nivel de & & & & & \\
\hline $\begin{array}{l}\text { impulso de los valores que } \\
\text { se dan desde el currículo }\end{array}$ & Encuesta dirigida a las y los & 1.- ¿El currículo de la carrera & A. Mucho & 17 & $85 \%$ \\
\hline universitario en función de & & impulsa el desarrollo de los valores? & $\begin{array}{l}\text { B. Poco } \\
\text { C. Nada }\end{array}$ & $\begin{array}{l}3 \\
0\end{array}$ & $\begin{array}{c}15 \% \\
0\end{array}$ \\
\hline de los estudiantes. & & 2.- ¿Cómo se concreta desde el & A. Mucho & 14 & $70 \%$ \\
\hline & & Currículo Universitario la & B. Poco & 6 & $30 \%$ \\
\hline & & formación axiológica? & Nada & 0 & 0 \\
\hline & & 3.- ¿La educación en valores es & A. Mucho & 19 & $95 \%$ \\
\hline & & importante como parte del perfil del & B. Poco & 1 & $5 \%$ \\
\hline & & Profesional en Trabajo Social? & C. Nada & 0 & $0 \%$ \\
\hline
\end{tabular}

Entrevista dirigida a las 1.- ¿Conoce Ud. Si se impulsa la 1.- La Facultad de Trabajo Social forma profesionales competentes y Autoridades. $\quad$ práctica de los valores como parte humanistas, que impulsa la práctica de valores a través de las asignaturas de la de la formación Profesional de los carrera y que complementa con las asignaturas profesionales, entre ellas están estudiantes de la Facultad de la Etica y Valores que debe de tener todo (a) Trabajador (a) Social (a). Trabajo Social? 2.- La práctica de valores se la da como parte fundamental en la formación de los profesionales en Trabajo Social, ya que la carrera es humanista y su accionar es importante.

$\begin{array}{lll}\text { OBJETIVO } & \text { Encuesta dirigida a las y los } \begin{array}{l}1-i \text { Cree Usted que las familias } \\ \text { disfuncionales influyen en la } \\ \text { práctica de valores? }\end{array} & \begin{array}{l}\text { A. Si } \\ \text { B. NoCIFICO } \mathrm{N}^{\circ} \mathbf{2}\end{array} \\ \text { Estudiantes. }\end{array}$

A. $\stackrel{\mathrm{Si}}{\mathrm{No}}$

Determinar los problemas práctica de valores?

que presentan los

estudiantes que provienen

de familias disfuncionales Encuesta dirigida a los y las relacionándolo con los desajustes emocionales que provocan
2.- ¿Señale en qué rango se ubican las familias de los estudiantes? disfuncionalidad familiar provoca desajustes emocionales en los estudiantes?

Entrevista dirigida a las

Autoridades. 1 - ¿De qué manera influyen las familias disfuncionales en la práctica de valores?
3.- ¿Considera que la

\section{A. Muy organizadas \\ B. Organizadas \\ C. Medianamente organizadas \\ D. Desorganizadas}

A. $\mathrm{Si}$

B. No

1. En la conducta, la forma de ser $y$ actuar, en algunas ocasiones pueden ser violentos y en otras puede demostrar aislamiento absoluto del grupo.

2. En las actitudes y comportamientos que reflejan diversos estados de ánimo, debido a los problemas familiares que presentan en sus hogares.

3. Es relativo, muchas veces pese a tener esta categoría se fundamentan en grandes valores.

Establecer la influencia de los medios de comunicación en la práctica de valores y las relaciones interpersonales de los estudiantes en el proceso educativo.
2.- ¿El contexto social influye en la práctica de los valores de los estudiantes de la Facultad de

A. $\mathrm{Si}$

$\begin{array}{rr}118 & 79 \% \\ 29 & 29 \%\end{array}$

Encuesta dirigida a las y los Estudiantes.

Entrevista dirigida a las 1 Trabajo Social?

1. ¿Cómo influyen los medios 1.- En la conducta y comportamientos de cada individuo. de comunicación en la formación en 2.-. Las actitudes que adoptan están a la defensiva de todo lo que se presenta a valores de los estudiantes de la su alrededor. Facultad de Trabajo Social? 


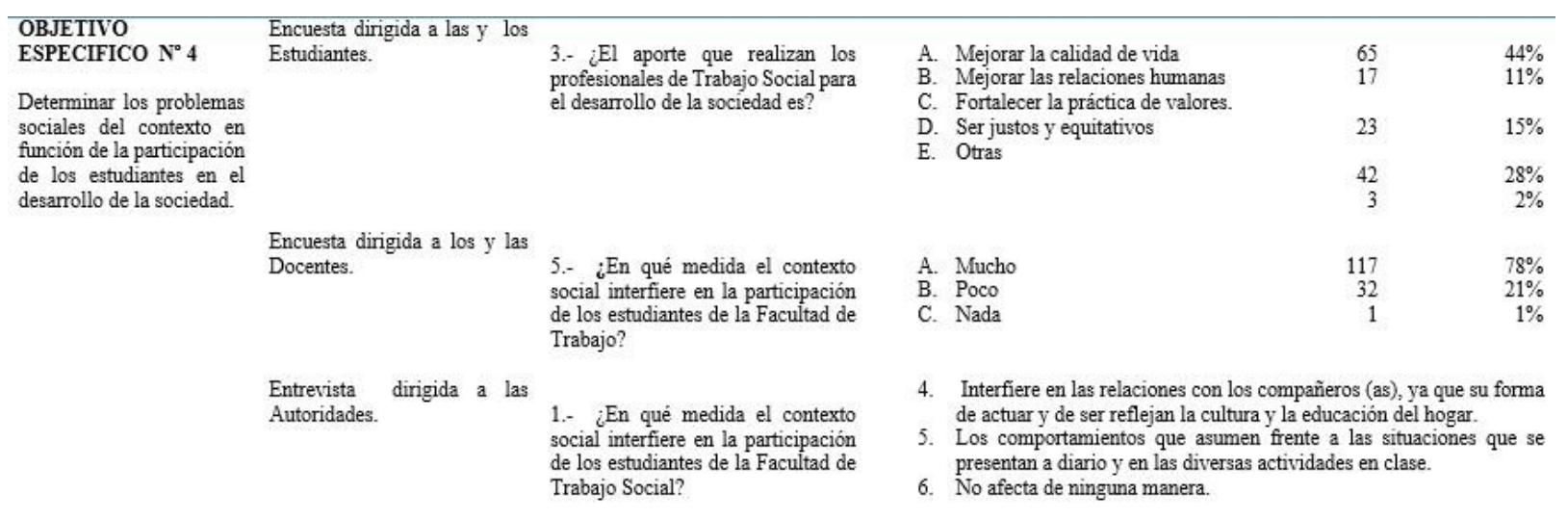

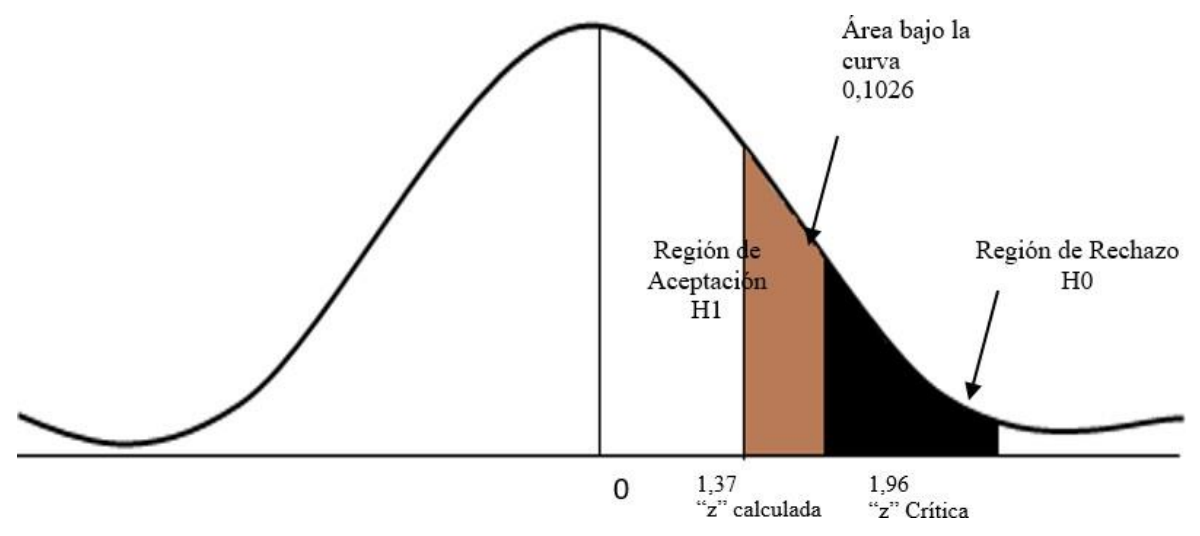

Grafico $N^{\circ}$ 1.- Validación estadística prueba de puntaje $Z$

\section{Conclusiones.}

El nivel de impulso de los valores que se promueven desde el currículo universitario es medianamente alto, siendo evidente la fundamental importancia en la formación en valores de los estudiantes de Trabajo Social, ya que la profesión es humanista, y es necesario fortalecer sus actitudes y/o comportamientos a través de las asignaturas de Ética y Valores que son parte de la 
Malla Curricular, y que se convierten en la parte fundamental del accionar de los Trabajadores Sociales, debido al rol que cumplen en el cambio en las relaciones humanas de la sociedad.

Los estudiantes de la Facultad de Trabajo Social que provienen de familias disfuncionales ha sido victimas de problemas internos y externos, debido a que no existe una sólida formación en valores por parte de los padres en el hogar, ya que con sus actitudes fomentan desajustes emocionales que perjudican el desarrollo y formación de la personalidad, provocando alteraciones en la convivencia educativa y social.

Los medios de Comunicación influyen negativamente en la práctica de valores de los estudiantes, ya que fomentan actitudes y comportamientos negativos, que los reflejan en la convivencia en el aula de clases, en ocasiones presentan un ambiente de hostilidad y resentimientos, es decir, no existen buenas relaciones interpersonales por el escaso manejo de las habilidades sociales en el proceso educativo.

Los problemas que más interfieren en la práctica del Trabajo Social son: la desintegración familiar debido a que por la situación económica difícil en la que viven, se ven obligados a viajar para buscar nuevas fuentes de trabajo y mejorar sus condiciones de vida, sin importar el precio que tienen que pagar, pues la consecuencia es una familia desintegrada y por ende hijos olvidados y abandonados a la voluntad propia, siendo vulnerables a las adicciones y problemas sociales; además se evidencia violencia intrafamiliar, todo lo antes mencionado interfiere en las relaciones con los compañeros (as). 


\section{Bibliografía.}

1) ALVARADO, M. La educación en valores del estudiante universitario a través del proceso docente - educativo. La Habana. (2000).

2) ARRIARÁN, Samuel. "Virtudes, valores y educación moral” (1999). Col. Textos. No 12. México. UPN.

3) BÁXTER E. y otros. La escuela y los problemas de la formación del hombre. Instituto Central de Ciencias Pedagógicas. La Habana. Cuba. 1994.

4) Cortina, A. (1998). El mundo de los valores. Ética mínima y educación. Ed. El Búho. Sta. Fé de Bogotá.

5) LUCINI, F. Educación en valores y reforma educativa. Revista Vela Mayor. Año 1, No. 2, Anaya Educación, España. (1994)

6) LEVIN, Michel. "La enseñanza de la ética" (2000) México, Siglo XXI. 